\title{
LES CARACTERISTIQUES FAUNIQUES DU NEOGENE DES DARDANELLES
}

\author{
Pax \\ Fikret OZANSOY
}

Ce travail a pour but d'indiquer les caractèrs palcontologiques et la succession faunique du Miocène terminal et du Pliocène avancé dans les régions des Dardanelles et du Gúlpınar (Ayvacık).

Les resultats obtenus ont mis en évidence non seulement les bio. zones des faunes mammaliennes, mais aussi les biofacies des invertébrés sur la cote anatolienne de cette contréc, depuis le Miocène supericur jusque dans le Pliocène infericur (d'après la géchronologic française) et au cours de l'unité gécohronologie du Pliockne (selon la chronologie nord-americaine).

Les caractères biologiques de la succession des groupez $x$ manifestent d'une manière gentrale comme suit:

Les unités biostratigraphiques contiennent uniquement des fau-

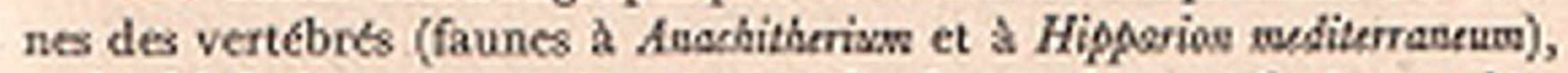
ou les biofacies sont representées par les faunes des vertébrés associées aux élements malacologiques (faune a Dorotberias et faune à cetotherien et par les faunes malcologieques (faunes à Mortra, a Ostrea).

Il s'agit d'une lutte entre les régimes continentaux (en pseticulier d'eau douce) d'une part et les régimes saumatres et marins d'autre part. Cette lutte se manifeste avec la prédominance du dernier régime marin au cours de la partie avancée de l'epoque du Pliocène dans la rìgion de Gülpınar, au Sud, dans la contré de Lápseki Çardak au Nord.

Du point de vue des caractères fauniques et de la superposition des unités biostratigraphiques, ce domaine paléontologique montre 
P'existance de ces différens régimes naturels qui sont de bas en hauts:

I) Regime continental I:

représenté par une faune mammalienne à Asckitkerium et à Listriadon splendens.

2) Regime continental II :

cette zone, dont la faune des vertébrés associce aux elements malacologiques d'eau douce est caractériséc également par ta présence de rares restes d'Fipparion, indique véritablement son régime naturel.

Malgré l'existance du genre Hipparion, il 's'agit d'une faune à Dorcathrium.

3) R6gime continental III:

faune malacologique d'eau douce.

4) Regime saumåtre: faune a Mortra I.

5) Regime marin:

faune malacologique marine et faunes a Cetotheriens.

6) Regime saumâtre:

faunes a Mortra II.

Les gisements fossiliferes, dans les régions des Dardanelles et Gülpanar (Ayvackk), sont représenteş par les deux biofacies principales.

La première est la zone inférieure qui est pardessus par la couche malacologique marine ou par la biozone aux cetotheriens. L'autre, est les gisements superieurs qui se manifestent bien apres la biozone préeddente. Mais dans la contrée de Gülpınar, cette deuxième zone saumattre sont representes par les innombrables couches successives qui nous ont fourni les faunes à Mastra. Il me sembleque l'on peut les grouper en une seul biozone relativement èpaisse.

7) Régime continental:

faune mammalienne à Hipporion oudilesroveuv (Gülpunar).

8) Regime marin:

faune à Ostrea (Guilpinar).

Comme on voit, les stdiments de l'époque du Neogène qui comprennent des unités biozonales bien importantes, ne se manifestent pas 
seulement tout le long des Dardanelles mais également au Sud de de cette region (Ayvacik-GULPINAR).

En outre, cette veritable succession faunique est trés impontante non seulement pour la Turquie mais aussi pour les macro-régions de Thetis et Parathétis.

Les recherehes effectúes plus récemmemt dans les régions etudiées ont permis de préciser les unités des biozones ainsi que eelles géchronologiques depuis le Mioctne siperieur jusque audessus le Pontien classique (Pikermien), c'est à dire juque dans la zone à Ostrea.

En effet, nous sommes en présence de la limite du Miocène-Pliocène dans la contróe des Daranelles. Mais il est encore difficile d'indiquer la véritable bande de limite.

Si, avec la chronologie nord - américaine qui fait debuter l'Łpoque du Pliocène avee l'apparition du genre Hipprion, on admet qu' à partir de la biozone a Dorcatberixm (ze zone) tous les gisements fossiliferes supéricurs doivent etre considétés comme les caractéristiques pour les temps pliocènes. En ce cas, les sédimens du Pliocène ont commencé a se développer, dans les bassins de tethis, paratethis, avant la zone de la vie a Dorrathrima (2 e zone) d'une part et l'époque du Pliccène sera beaucoup plus longue que croit d'autre part. s, avec l'école française, on admet que la biozone pikermienne classique de Gülpunar appartient au Miocene terminal, toutes les biozones representent les sous-unités géochronologiques superieures du Miocène, sauf la dernière zone à Osfres ( 7 e zone). Je n'ose pas discuter sur ces deux questions. Mais, au point de vue (pattern) géologique les régions étudiées et leurs sédiments aux biozones sont trés impontants.

I'importance principale de la sucoession faunique indique d'abord la présence de différents régimes naturels successifs dans la metme rogion, puis, la biozone a la faune marine (c'est ì dire je zone) donne, encore une fois, de la grande impontance à cette contrée.

Cette importance de la zone a faune marine consiste dans le fait qu'elle témoigne d'une transgression visible de Théts-parathétis sur la venue entre les deux aiges de deux biozones continentales a Hipparion (zones 2 et 7 ). 


\section{Les regimes et les biozones geographiques}

Comme on voit sur le tableau I, les regimes sont representés par leurs zones fossiliferes. En $\propto$ cas, nous avons deja 8 biozones successives, comme suit:

Zone I: faune à Auchitóriom (Bayrak Tepe)

Zone II: faune à Dorcatherium (et à Hipparias (Bayrak Tepe)

Zone III : faune malacologique d'eau douce (Bayrak Tepe)

Zone IV: faune aे Mactra I (Intepe-Kiraz pinar)

Zone V: faune malacologique (marine) (Intepe-Kiraz Pınar) et faune aे cetotherien (Bayrak tepe, Eski Subaşi)

Zone VI : faune a Mactra II (Kiraz Pınar, Barak Tepe, Gul Pınar, B. Subaşi)

Zone VII: faune a Hipharion mediterrasexos (Gul Pinar)

Zone VIII: faune a Ostrea (Gül Pinar)

Les sediments du Néogène des Dardanelles (Canakkale-IntepeLApseki et Cardak) comprennent des biozones de régimes I, II, III, IV et $\mathrm{V}$ et les couches fossiliferes de Gulpmar (Külahltayağa) appartiennent aux régimes VI, VII et VIII. (voir tabl. I).

\section{Zones de Bayrak Tepe (Mont Drapean)}

(Zones, I, II, III, V et VI)

Le Bayrak Tepe est situé a $5^{-6}$ kilomètre environs au Sud de la la ville de CANAKKALE.

Cette coline est haute, large et sa partic occidentale est coupée localement par des vallons courts, dans lesquels on peut suivre les couches fassiliféres inférieures qui comprennent des zones, ci-dessus mentionneses.

Au point de vue litostratigraphique et de la biofacies, le Bayrak, Tepe forme une partic saillante d'une formation principale qui s'etant tout au long de detroits de Chanakkale. Elle se rencontre également sur la cote de la Mer Egée septentrionaele, à l'Ouest d'Ayvacik (GULPINAR-Source de Roee). Ici on observe la partie supérieure des couches 
Dardandiles et Gülpinar (Aywack)

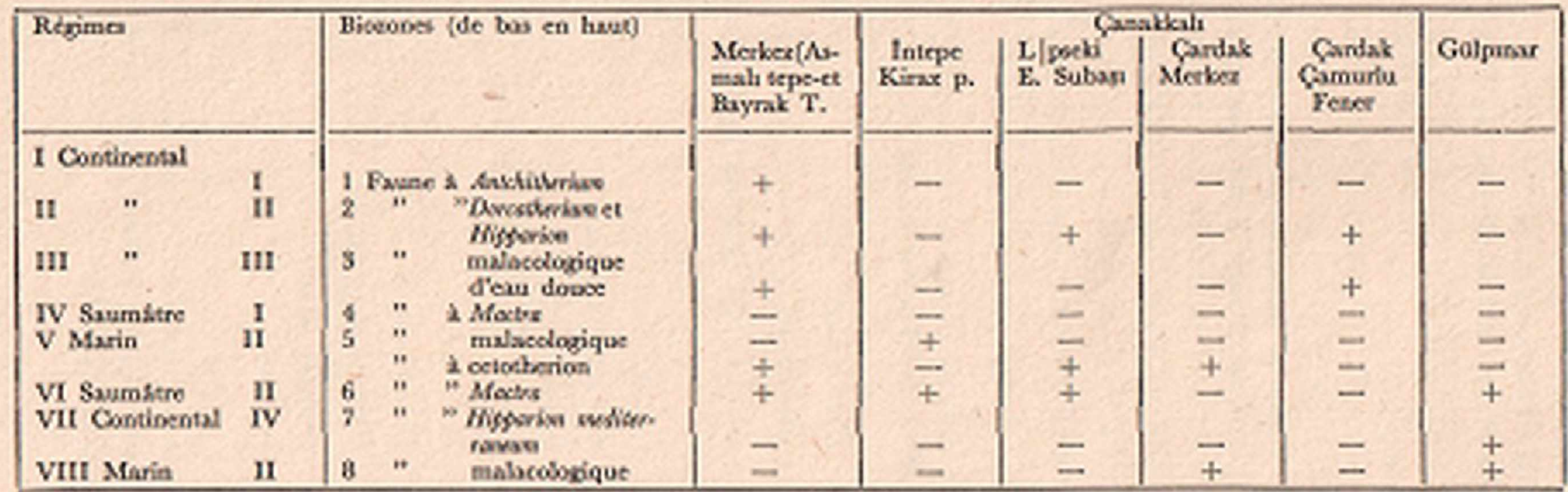


appartenant aux vallons de Bayrak Tepe qui montre un faciés d'eau saumitre surmonté d'abord par un complexe continental de Gül. panar à la zone de faune mammalienne de Pikermi.

Je propose de designer cette vaste deposition sous le nome de formation des Dordaselles.

\section{Zone $\mathrm{I}$ :}

Un niveau se comprase de sables blancs, legerment verdattre, alternant avec de conglomierats rouge ì élèments petits et à stratification entrecroisbe. Cette zone fossilière se trouve dans le niveau supérieur formé de sables qui est surmontee par la conglomerat la plus supéricure (de la mème horizon).

Il est possible de diviser la pecmiène zone fossiliére en deux partic:

1) zone I inf: forme de sables

2) zone I sup.: forméc de conglomerats rouges sup. Elles nous ont fourni les restes des vertebrés suivantes:

Proboscidea : Dinukerium? sp. Trilsphodst? sp.

Perissodactyla: Anofitherixk (grande taille) (voir Ozansoy, 1969) Rhinoceros sp.

Artiodactyla : Dercateritum sp.

Listriadon spleodors

Listrodas sp. (Busblistridae)

Gozella sp. (forme guadigi)

Carnivora : Hyenide

Mustclidé

Carnivore gen. sp. indet.

Reptilia : : texdudinides reptiles

Il s'agie d'une faune curasiatique. Elle comprent en partie les éléments des faunes mammaliennes du Miscène et du Pliocène de l'Anatolic et Thrace dans les mème genres, tels que Listrodas, ANchitberiam, Dorcatherima, Gazdla. 
Il y a quelques années, on a decouvert également en Anatolic les deux biozones qui on fourni les faunes ì Anstitherixu. Line associce au genre Hipparion et la majorité de son cortège (Ozansoy, 1969), l'autre faune à Arehithorivo posséde le caractère du Miocéne suptrieur (Sickenberg et Tobien, 197r).

La biozone de cette dernière faune à Anchitherism (Anchitherisms depetite taillc) qui se manifeste dans la règion d'Ankara, Kalecik-Candır- Hırsaz Dere (Ruisseau de Cambrioleur), doit être mis en parallèle avoc une partic au moins de l'unité géoclưonologique de la zone I de Bayrak Tepe.

Toutes les deux faunes mammaliennes indiquent veritablement, en Anatolie, la presence de caractères biologiques principaux (genetiques, systematiques, evolutifs ainsi que paléocoologiques), des faunes pliocène bien avant le Pliocène.

\section{Zone II:}

Dans la méme asxise (Bayrak Tepe), sur le versan SW, une couche formó de sabes bruns repose sur ic niveau à la faune à Anohithriar (de grande taille).

La zone fossilifere nous a fourni la faune des vertebres suivate': Probscidea : Probascidien gen. et sp. indet.

Perissadactyla: Fipparion sp. (reste rare)

Dikerass sp. (Sen)

Artiodcyla : Gazello sp.

Antilope gen. et sp. indet.

Sasorkeriam sp. (forme "pamiri")

Palaestragus sp.

Dorcatkerime sp.

Sus sp. (forme "mojor")

Carnivora : Hyénidés

Intilbriams sp.

Mustelidés

Melinés

: Strmofiber sp. 
Pinnipedia : Phose sp.

Cetacea : Cetotherien (Cetothorisw?)

Reptilis : tesdudinides

Pices : poissons

Invertebrata : invertebres (mollugques d'eau douce)

L'importanœe de cette faune des vertebres est considernbu, ar elle se trouve, d'abord, dans le domaine palbontologique d'une région de contact de Thétis-Parathétis, en suite elle nous montre la plus ancienne faune à Hipperion de Turquie dans un véritable échelle biostratigraphique. Bn effet, la faune des vertebrés, ci-haut mentionnec, est limite en bas par la faune à Aothitherixos (faunule continentale) et en haut par la biozone à celtotherien et par les couches à Mactre. Et dernièrement, il s'agit d'une biozone moyenne qui relie la véri. table faunule pikermienne ì celle du Miockne.

\section{Unite geochronologique}

Si P'on fait debuter le Pliocène avec l'apparition du genre Hipparion (Ozansoy, 1957. Arambourg, t959), cette biozone appartient au Pliocène (Plicoène inférieur, posterieur à la biozone classique de de Clarandon). Mais au point de vue geologique, si l'on peut étre considertes les couches marines supérieures, ce gisement foosilifere et sa zone appartiennent au sommet superieur du Miocéne supérieur.

\section{Zone III:}

Gisement formé de sables. Il contient une faune des invertebrés d'eau douce.

L'importance de cette biozone consiste dans le fait qu'elle nous indique la continuation du régime d'eau douce.

\section{Zones IV-VI:}

Elles se compasent de caleaires, légeremenet bruns, alternant avec des sables et des argiles. Elles comprennent des biozones au caracteres marins (saumattre-marin).

Les niveaux de ces zones s'etadent tout le long de detrois de Çanak. 
kale et se manifeste en partie dans la region d' Ege septentrionale (Ayvacık-Golpinar).

Cet assixe (zones IV-VI) comprend des couches successives a $M$ act$\mathrm{ra}$, a cetotheriens et a la faune malacologique bien marine.

La disposition de ces biofacies $x$ suite dans une phase relativement transgressive et ceci peut indiquer que les influences de regimes marins été sensibles dans cette region. Il me semble qu'il est possible de chercher la limite du Miocene-Pliocene dans cette assise. On nela trouve que cependant e long de detrois de çanakkale et sur la cotod Ege septentrionale: elle est proprement tethisien- paratethisien

\section{Zone VII:}

Domine par une serie de tuffs. Elle se manifeste dans la region d'Ayvacik (Gulpınar-Külahhayăg). Cette zone comprend d'une fane mammlienne a Hiparrios suediterranerm.

D'après nos rocherches palcontologiques, le developpement de continent d'Anadolic, depuit l'epogue de l'Oligocene, a favorise en particulier les faunes mammaliennes a hipourion parmi. La majorite de ces faunes est representees par la presence $\mathrm{d}^{\prime} H$. nudifmanrom.

Au point de vue de l'expansion, la biozone de Gülpunar nous a fourni cette faune a $H$. meditorrasesw. Ciest une faunule la plus occiden. tale de l'Anatolic:

\begin{tabular}{|c|c|}
\hline Carnivora & $\begin{array}{l}\text { Ictitsoriam sp. } \\
\text { Hyansa so. }\end{array}$ \\
\hline Proboscidea & $\begin{array}{l}\text { Probascidien de grande taille } \\
\text { (Sycosnolopbess ?) } \\
\text { Chossoloptdos sp. (pentelici) }\end{array}$ \\
\hline Perissodactyla & 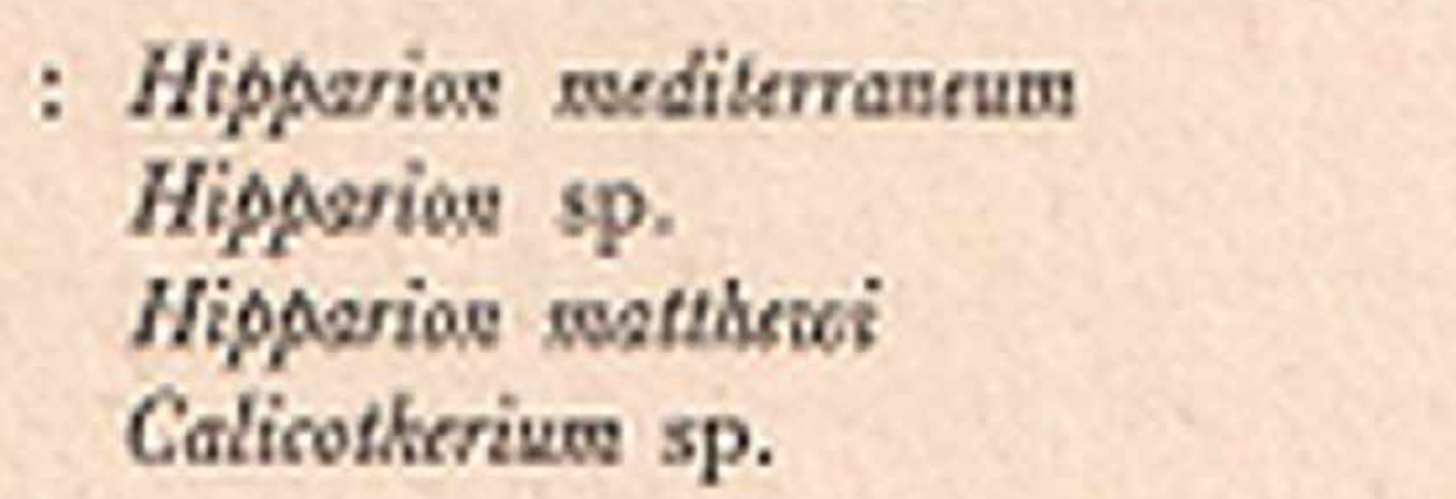 \\
\hline Artiodactyla & $\begin{array}{l}\text { Sus sp. } \\
\text { Palowtragus sp. } \\
\text { Senstlorian sp. }\end{array}$ \\
\hline
\end{tabular}




\section{Helladothrises sp. \\ Antilope gen. sp. indet. \\ gazella sp.}

Ce facies est pardessus par les couches a la zone VIII.

Entre la zone VII ct le niveau des zones IV-VI, il existe un diasteme stratigraphique qui est represente d'abord par un conglomerats, puis par une surface d'erosion.

Si l'on fait debuter l'epoque du Pliocee avec le commencement de la zone IV (ou bien avec la cracteristique faunique de la biozone IV) dans les regions d'Ege septentrional, des Dardanelles et meme dans la region de la mer de Marmars, la zone VII et sa faune appartiennent au sommet du Pliocene inferieur. En ce cas, la zone II (faune a $D$ w'athrius et a Hoppartsi) apparticnt au Miocene terminal ?.

\section{Zone VIII:}

Zone caracterisce par une bien marine (faune a Ostres)

Une epoyue transgressive tres sensible est representec das la region de Gülpmar par cette biozone.

C'est une biozone tres importante. Car, elle nous explique d'abord nouvelle epoque (on qous-cpope) trangressive de moins au Sud des Dardanelles (peut-etre dans une grande region) bien posterieur a la zone de la vic a Hippariou medikerouese. En outre, elle nous indique egaleme nt yu'il n'y ait cu plus des migrations terrestres, en particulier des faunes a Hipparion, de l'Est vers l'Ouest vers l'Est. 